\title{
Heat Treatment of Duplex Stainless Steel 2205 by Inserting Nano Nd2FeB14 in HIP Manifolds Under the Scope of Category Theory
}

\author{
Mohamed Atef Mohamed Gebril ${ }^{1}$ \\ ${ }^{1}$ Department of material engineering, Faculty of petroleum and minning engineering, Suez University, Egypt \\ Correspondence: Mohamed Atef Mohamed Gebril, Department of material engineering, Faculty of petroleum and \\ minning engineering, Suez University, Egypt. E-mail: atefmohamed840@yahoo.com
}

Received: September 14, 2016

Accepted: September 23, 2016

Online Published: October 21, 2016

doi:10.5539/jmsr.v6n1p1

URL: http://dx.doi.org/10.5539/jmsr.v6n1p1

\begin{abstract}
In this paper, improving mechanical properties of duplex stainless steel by spinodal reaction inhibitors has been discussed. Spinodal gaps can be minimized when the host particles are minimized to the nanoscale. The Cahn-Hilliard Equation and the Allen-Cahn Equation on Manifolds with Conical Singularities can be related to manifolds produced by hot isostatic pressing (PM HIP) are used to improve mechanical strength. Spinodal reactions will be Inhibited by magnetic treatment and Nd-Fe-B Magnets. Grain refiners will be used to retard spinodal reactions. Mathematically, category theory is used to establish links between these different concepts.
\end{abstract}

Keywords: Nd2Fe B14 magnetic thin films, Duplex stainless steel 2205, Impact energy, spinodal reactions, cahn hillard equations, Ising model, Ohta-Kawasaki energy, Fractals - solobev space, Finsler manifold, The Lusternik-Schnirelman category

\section{Introduction}

Duplex stainless steels are called "duplex" because they have a two-phase microstructure consisting of grains of ferritic and austeniticstainlesssteel.Duplexstainlesssteelshavebeenincreasinglyusedfor a variety of applications in marine construction, chemical industries and power plants due to their excellent combinational of mechanical properties and corrosion resistance The superior properties of duplex stainless steels come primarily from approximately equivalent amounts of austenite $(\gamma)$ and $\delta$. It is well known that in $\mathrm{Fe}-$ Cralloys, there is a miscibility gap, where the ferrite phase may decompose into an iron-rich BCC phase $(\alpha)$ and a chromium-enriched BCC phase $\left(\alpha^{\prime}\right)$ by spinodal decomposition the effect of isothermal treatment temperatures ranging between (400and500C) on the embrittlement of a2205duplex stainless steel (with $45 \%$ ferrite-55\% austenite, vol.\%) has been investigated. The impact toughness and hardness of the aged specimens were measured. The results show that the steel is susceptible to severe embrittlement when exposed at $475 \mathrm{C}$. High-resolution transmission electron microscopy has revealed that anisotropic spinodal decomposition occurred in the steels during aging at $475 \mathrm{C}$; the $\delta$-ferrite decomposed into a nanometer-scaled modulated structure with a complex interconnected network, which contained a Fe-rich BCC phase $(\alpha)$ and $\mathrm{Cr}$-enriched BCC phase $\left(\alpha^{\prime}\right)$.It is deduced that the locking of dislocations in the modulated structure leads to the severe embrittlement. Spinodal reactions are retarded by inserting nanoparticles in certain places. Also, we can reduce these spinodal reactions by grain refiners as they will be used to modify gibbs free energy for the total systems. By applying magnetic field and making magnetic bubbles, we can use their properties to control in the thermal charachteristics of the entire system (Alvarez-Armas \& Degallaix-Moreuil, 2009; Adams, Olubambi, Potgieter, \& Van Der Merwe, 2010; Kangas \& Newman, 1998).

Permanent magnet is a ferromagnetic material that has a magnetization even if there is no external applied field. Using Ising model we can explain their properties, and the Ising model has special charchteristic in manifolds. Nd-Fe-B magnets has special properties, Fractal Magnetic Domains on Multiple Length Scales in Nd2Fe14BThe industrial strength ferromagnet, $\mathrm{Nd} 2 \mathrm{Fe} 14 \mathrm{~B}$ has become a prototypical system for the study of magneticdomain structures. Below the Curie temperature, Tc $1 / 4565 \mathrm{~K}$, the Nd and Fe moments order ferromagnetically (Byrne, Spence, Olsen, Houghton, \& MCMAHON, 1994).

Nowadays, many companies use HIP thechniques to create manifolds to enhance mixed (mechanical and electrochemical ) properties. Also, we will study the effect of nano particles in the spinodal gap and their strong relation to cahn -callihard spinodal reactions. 
Many theories in the group theory are used to describe the actions of group, in addition, functors and classes in the category theory are used to establish many beautiful relations between different mathematical structures (Bucur, Deleanu, \& Hilton, 1968; May, 1999).

The paper is organized as follow. In section 2, properties of duplex stainless steel were briefly reviewed. In section 3 , Concepts in the theory of categories were briefly discussed. In section 4 , many experimental data are collected to achieve progress in the development of the properties of duplex steel. In section 5, we have mentioned the results and the discussion how to develop duplex stainless steel using mathematical theories. The work closes with some concluding remarks in section 6 .

\section{Duplex Stainless Steel}

Duplex stainless steels are becoming more common. They are being offered by all the major stainless steel mills for a number of reasons:

- Higher strength leading to weight saving

- Greater corrosion resistance particularly stress corrosion cracking

- Better price stability

- Lower price

There is a conference on the subject of duplex every 2-3 years where dozens of highly technical papers are presented. There is a lot of marketing activity surrounding these grades. New grades are being announced frequently.

Yet, even with all this interest, the best estimates for global market share for duplex are between 1 and 3\%. The purpose of this article is to provide a straightforward guide to this steel type. The advantages and disadvantages will be described.

Ferritic - low strength (a bit higher than austenitic, $250 \mathrm{MPa} 0.2 \% \mathrm{PS}$ ), poor weldability in thick sections, poor low temperature toughness. In addition, the high nickel content of the austenitic types leads to price volatility which is unwelcome to many end users. The basic idea of duplex is to produce a chemical composition that leads to an approximately equal mixture of ferrite and austenite. This balance of phases provides the following:

- Higher strength - The range of $0.2 \%$ PS for the current duplex grades is from $400-550 \mathrm{MPa}$. This can lead to reduced section thicknesses and therefore to reduced weight. This advantage is particularly significant for applications such as:

o Pressure Vessels and Storage Tanks

o Structural Applications e.g. bridges

- Good weldability in thick sections - Not as straightforward as austenitics but much better than ferritics.

- Good toughness - Much better than ferritics particularly at low temperature, typically down to minus $50 \mathrm{C}$, stretching to minus $80 \mathrm{C}$

Duplex 2205 is a nitrogen enhanced duplex stainless steel that was developed to combat common corrosion problems encountered with the 300 series stainless steels. "Duplex" describes a family of stainless steels that are neither fully austenitic, like 304 stainless, nor purely ferritic, like 430 stainless. The structure of 2205 duplex stainless steel consists of austenite pools surrounded by a continuous ferrite phase. In the annealed condition, 2205 contains approximately $40-50 \%$ ferrite. Often referred to as the work horse grade, 2205 is the most widely used grade in the duplex family of stainless steels.

It is well known that in $\mathrm{Fe}-\mathrm{Cr}$ alloys, there is a miscibility gap, where the ferrite phase may decompose into an iron-rich BCC phase $(\alpha)$ and a chromium-enriched BCC phase $\left(\alpha^{\prime}\right)$ by spinodal decomposition the effect of isothermal treatment temperatures ranging between $(400$ and 500C) on the embrittlement of a2205duplex stainless steel (with $45 \%$ ferrite- $55 \%$ austenite, vol.\%) has been investigated. The impact toughness and hardness of the aged specimens were measured. The results show that the steel is susceptible to severe embrittlement when exposed at $475 \mathrm{C}$. It is deduced that the locking of dislocations in the modulated structure leads to the severe embrittlement. Spinodal reactions are retarded by inserting nanoparticles in certain places (Alvarez-Armas \& Degallaix-Moreuil, 2009; Hsieh, Tsai, Chang, \& Yang, n.d.; Huang \& Shih, 2005).

\section{Concepts in the Theory of Categories}

Category theory formalizes mathematical structure and its concepts in terms of a collection of objects and of arrows (also called morphisms). A category has two basic properties: the ability to compose the arrows 
associatively and the existence of an identity arrow for each object. The language of category theory has been used to formalize concepts of other high-level abstractions such as sets, rings, and groups.

Several terms used in category theory, including the term "morphism", are used differently from their uses in the rest of mathematics. In category theory, morphisms obey conditions specific to category theory itself.

Samuel Eilenberg and Saunders Mac Lane introduced the concepts of categories,functors, and natural transformations in 1942-45 in their study of algebraic topology, with the goal of understanding the processes that preserve mathematical structure.

Category theory has practical applications in programming language theory, in particular for the study of monads in functional programming.

A category $C$ consists of the following three mathematical entities:

- A class ob $(C)$, whose elements are called objects;

- A class hom $(C)$, whose elements are called morphisms or maps or arrows. Each morphism $f$ has a source object $\boldsymbol{a}$ and target object $\boldsymbol{b}$.

The expression $f: a \rightarrow b$, would be verbally stated as " $f$ is a morphism from $a$ to $b$ ".

The expression $\operatorname{hom}(\boldsymbol{a}, \boldsymbol{b})$ - alternatively expressed as $\operatorname{hom}_{c}(\boldsymbol{a}, \boldsymbol{b}), \operatorname{mor}(\boldsymbol{a}, \boldsymbol{b})$, or $\boldsymbol{C}(\boldsymbol{a}, \boldsymbol{b})$ - denotes the hom-class of all morphisms from $a$ to $b$.

- A binary operation $\circ$, called composition of morphisms, such that for any three objects $a, b$, and $c$, we havehom $(b, c) \times \operatorname{hom}(a, b) \rightarrow \operatorname{hom}(a, c)$. The composition of $f: a \rightarrow b$ and $g: b \rightarrow c$ is written as $g \circ f$ or $g f$, governed by two axioms (Kim et al., 2005):

Associativity: If $f: a \rightarrow b, g: b \rightarrow c$ and $h: c \rightarrow d$ then $h \circ(g \circ f)=(h \circ g) \circ f$, and

Identity: For every object $x$, there exists a morphism $1_{x}: x \rightarrow x$ called the identity morphism for $x$, such that for every morphism $f: a \rightarrow b$, we have $1_{b} \circ f=f=f \circ 1_{a}$.

From the axioms, it can be proved that there is exactly one identity morphism for every object. Some authors deviate from the definition just given by identifying each object with its identity morphism (Kreyssig et al., 2009; Borceux, 1994; May, 1999).

\section{Experimental Procedure and Methods}

1. The chemical composition of wrought 2205 duplex steel studied was Fe-22.62Cr-5.12Ni-3.24Mo-1.47Mn $-0.38 \mathrm{Si}-0.02 \mathrm{C}$ (wt\%). The as-received 2205 duplexstainless steel rods for this research was produced by Gloria Material Technology Corporation through the 4-folded forging of a cast slab at $1160-1180^{\circ} \mathrm{C}$ and annealing at $1050^{\circ} \mathrm{C}$ for $30 \mathrm{~min}$, followed by water quenching. The annealed steel gave a dual-phase structure (with $45 \delta$-ferrite -55 austenite, vol\%) without other secondary phases. Experiments in this report contained two parts. The first part was to investigate the microstructure of the unaged and aged specimens without deformation, including dislocation characterization and dislocation density calculation. The second part focused on the evolution of microstructures and dislocations in the unaged and aged specimens after Charpy impact test. In the first experiment, specimens $6 \mathrm{~mm}$ in length and $3 \mathrm{~mm}$ in diameter were machined from the steel bar and aged at $475^{\circ} \mathrm{C}$ for $64 \mathrm{~h}$. Optical microscopy specimens sliced from the aged specimens were mechanically polished and then electrically etched in $10 \mathrm{~N} \mathrm{NaOH}$ solution at 9V. Transmission electron microscopy specimens were also sliced from the aged specimens, thinned to $0.06 \mathrm{~mm}$ by abrasion on $\mathrm{SiC}$ papers and twin-jet electropolished using a mixture of $5 \%$ perchloric acid, $25 \%$ glycerol, and $70 \%$ ethanol in the temperature range of -5 to $-10^{\circ} \mathrm{C}$ and $40 \mathrm{~V}$ etching potential. The microstructures and microanalyses of the specimens were investigated using a FEI Tecnai G2 T20 transmission mission electron microscopy. In the second experiment, Charpy specimens in which the elongated austenite grains were perpendicular to V-notch were machined in the standard $10 \mathrm{~mm} \times 10 \mathrm{~mm} \times 55 \mathrm{~mm}$ dimensions and aged at $475^{\circ} \mathrm{C}$ for $64 \mathrm{~h}$.

After the aging treatment, Charpy impact tests were carried out at room temperature and the specimens were sliced longitudinally for optical microscopy and scanning electron microscopy observation.

To avoid the interference from deformation, the unaged and aged specimens were observed without impact test first. Fig. 1a and Fig. $1 \mathrm{~b}$ show the OM of the unaged and aged specimens in transverse sections, from which and no difference between their microstructure can be found. Elongated austenite grains showed in Figure 1c. shows the macrographs of the specimens after the Charpy impact tests. The unaged specimen in Figure 3a was heavily deformed with rough failure surface and the impact energy was 278J (Hsieh, Tsai, Chang, \& Yang, n.d.). 

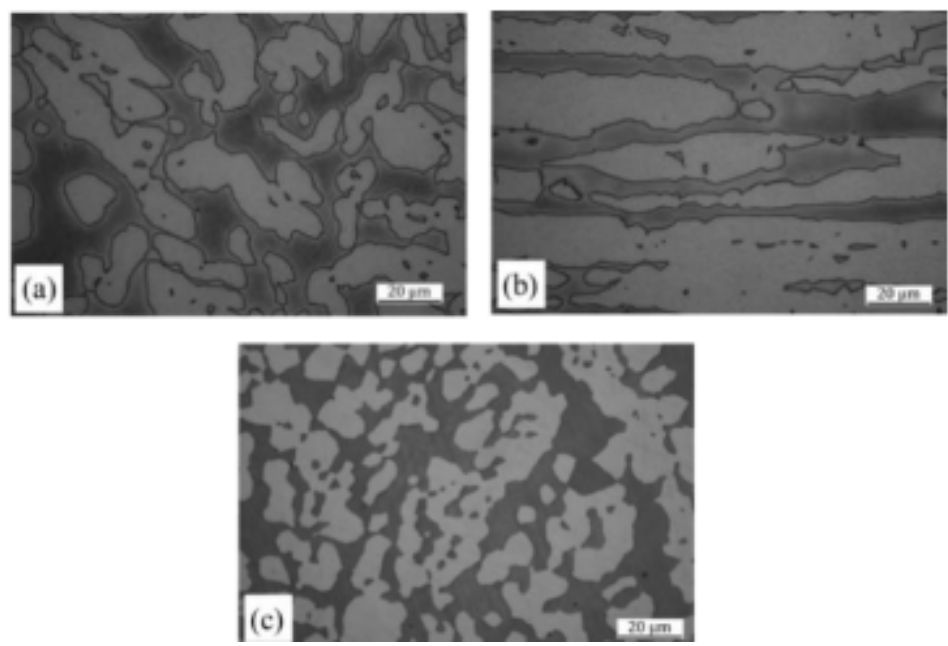

Figure 1. Optical micrographs of the 2205 duplex stainless steel bar: (a) transverse section of the unaged specimen; (b) longitudinal section of the unaged specimen; (c) transverse section of the aged specimen

Table 1. Dislocation densities and average lengths of the unaged and aged $\delta$-ferrite grains

\begin{tabular}{lll}
\hline & Dislocation density $\left(\mathrm{m}^{-2}\right)$ & Dislocation length $(\mathrm{nm})$ \\
\hline Unaged $\delta$-ferrite & $8.3 \pm 2.1 \times 10^{14}$ & $234 \pm 23$ \\
Aged $\delta$-ferrite & $8.2 \pm 2.5 \times 10^{14}$ & $262 \pm 36$ \\
\hline
\end{tabular}
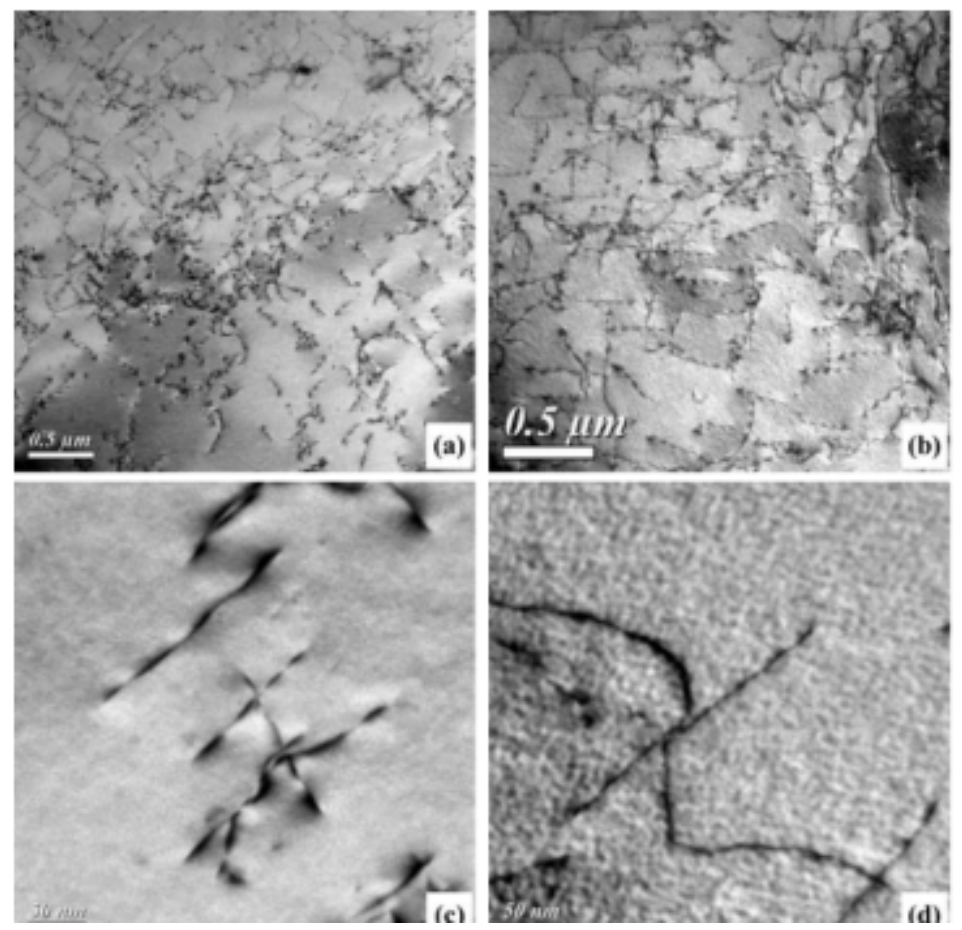

Figure 2. Transmission electron micrographs of the: (a) unaged $\delta$-ferrite; (b) aged $\delta$-ferrite; (c) unaged $\delta$-ferrite with high magnification; (d) aged $\delta$-ferrite with higher magnification 
2. Probing Fractal Magnetic Domains on Multiple Length Scales in Nd2Fe14BThe industrial strength ferromagnet, $\mathrm{Nd} 2 \mathrm{Fe} 14 \mathrm{~B}$ has become a prototypical system for the study of magneticdomain structures. Below the Curie temperature, $\mathrm{Tc} 1 / 4565 \mathrm{~K}$ the $\mathrm{Nd}$ and $\mathrm{Fe}$ moments order ferromagnetically. The crystal-electric field produces a strong magnetic anisotropy with the easy axis along the tetragonal c direction. Below the spin-reorientation temperature, TSR $1 / 4135 \mathrm{~K}$, the magnetic structure (and easy axis direction) changes via a second order transition and becomes conelike in which the moments are canted away from the $\mathrm{c}$ direction by an angle that increases from 0 at T SR to 28 at $4 \mathrm{k}$. The moments lie in one of the four symmetry-equivalent $\mathrm{f} 110 \mathrm{~g}$ planes in agreement with calculations of crystal-electric field effects. A multitude of techniques have been used to image magnetic domains at exposed surfaces of $\mathrm{Nd} 2 \mathrm{Fe} 14 \mathrm{~B}$, such as Bitter decoration, Kerr microscopy, Lorentz [and holographic transmission electron microscopy, scanning electron, and magnetic force microscopy. For imaged surfaces perpendicular to the $\mathrm{c}$ direction, domains have been observed with dimensions between $2-5 \mathrm{~m}$ at $4 \mathrm{~K}$ (Kim et al., 2005 ) and about 0:1-0:6 $\mathrm{m}$ at room temperature, respectively. These values are very close to the single domain size of about 0:2-0:4 m determined by magnetization measurements on polycrystalline samples manifest smooth, mirrored surfaces and can have volumes as large as $1 \mathrm{~cm}^{3}$ (Condette, Melcher, \& Süli, 2011; Kreyssig et al., 2009).

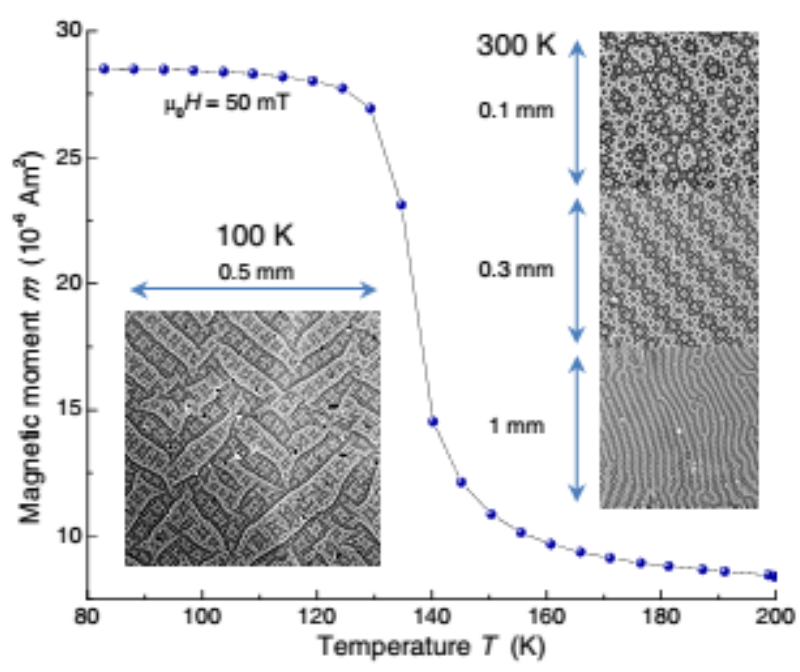

Figure 3. Temperature dependence of magnetization and the magnetic domain patterns $\mathrm{Nd} 2 \mathrm{Fe} 14 \mathrm{~B}$ single crystal. The magnetization was measured at $\mu_{0} H=50 \mathrm{mT}$ applied along $\mathrm{C}$ axis

3. conventional methods of manufacture of high pressure process manifolds in duplex stainless steelshaveemployed 3 basic approaches.

a) pipe and butt weld outlets

b)extruded branches(including non standardtees)

c)swept outlets.

These methods are all labour intensive manufacturing routes requiring the manufacture of usually, nonstandard seam less or welded pipe of thick section. This then has to be forged or machined prepared to accept the out let sort manifold fabrication. As such, these routes can be associated with high costs and long lead times. Also when duplex and super duplex stainless steel involved considerations of quality of fabrication, distortion due to residual stresses and ease of inspection and interpretation of ND Edatacan become problematical. Many of these difficulties can be avoided if the technique of Hot Isostatic Pressing (HIP) isused for the manufacture of near net shape manifold sections with integral branches and branch connections. Generally, HIPDISDSS can achieve 70 Joules average Charpy " $\mathrm{V}$ " notch impact test results at $-10^{\circ} \mathrm{C}$ which is commensurate with a conventional forging. However, the form of the full impact energy vs temperature transition curve is quite different, since HIP items do not exhibitan impact transition temperature like conventional wrought products, Figure 5. In this case HIP products mirror the behaviour of DISDSS weld metals, where the oxygen content lowers the upper shelf impact energy and extends its range to lower temperatures. This impact transition behavior of HIPD/SDSS is not currently 
recognised by design 1 fabrication codes which are real based up on the behavior of wrought ferritic or austenitic steels. Hence codes call for high (uppershelf) levels of charpy toughness at minimum design temperature or below dependent upon the thickness compensation factor (Byrne, Spence, Olsen, Houghton, \& MCMAHON, 1994; Park \& De Cooman, 2014; Udarne \& Nerjavnega, 2015).

4. By imserting nano Nd2FeB14 in the HIP manifolds of duplex stainless steel and then making analysis for dislocation denisity we find the following:

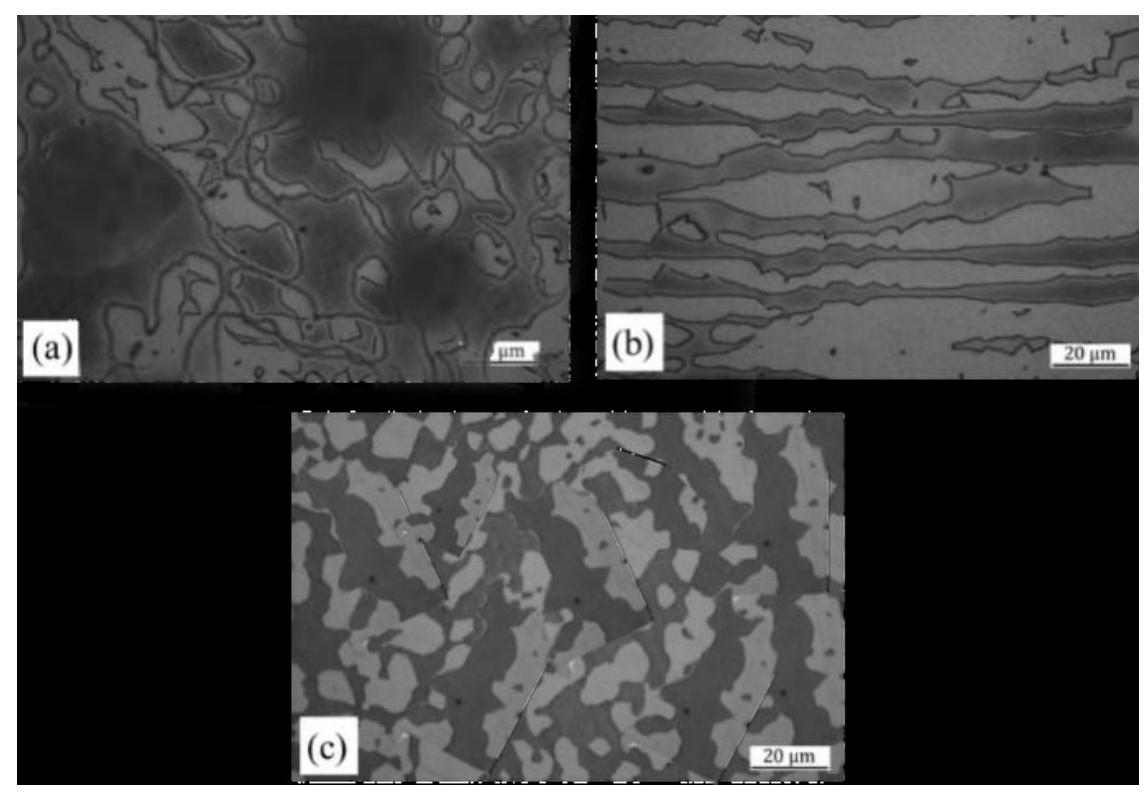

Figure 4. Optical micrographs of the 2205 duplex stainless steel bar after inserting Nd2Fe B14 particles in HIP manifold.(dislocation densities vary considerably) : (a) transverse section of the unaged specimen; (b) longitudinal section of the unaged specimen; (c) transverse section of the aged specimen

And also by applying high magnetic field we have enhanced impact energyat aging from $22-25 \mathrm{~J}$ to 45 to $100 \mathrm{~J}$ depends on the magnetic field as showed in the figure (Udarne \& Nerjavnega, 2015; Maehara, Koike, Fujino, \& Kunitake, 1983; Hsieh, Tsai, Chang, \& Yang, n.d.).

You can see high degree of brightness as we have applied high and intensive magnetic field (Maehara, Koike, Fujino, \& Kunitake, 1983; Park \& De Cooman, 2014; Frost \& Ashby, 1982; Hsieh, Tsai, Chang, \& Yang, n.d.).

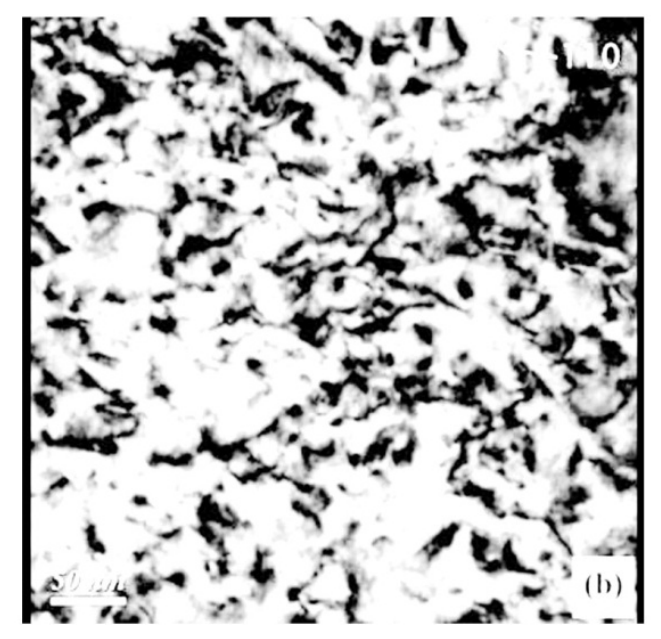

Figure 5. Aged $\delta$-ferrite grain after impact test after inserting Nd2 Fe B14 particles in HIP manifold and the application of high magnetic field 


\section{Results and Discussions}

In Duplex stainless steel 2205, spinodal reactions have bad effects in the mechanical properties of duplex stainless steel. The spinodal reactions have strong relations to the gibbs free energy (Alvarez-Armas \& Degallaix-Moreuil, 2009; Park \& De Cooman, 2014; Hsieh, Tsai, Chang, \& Yang, n.d.).

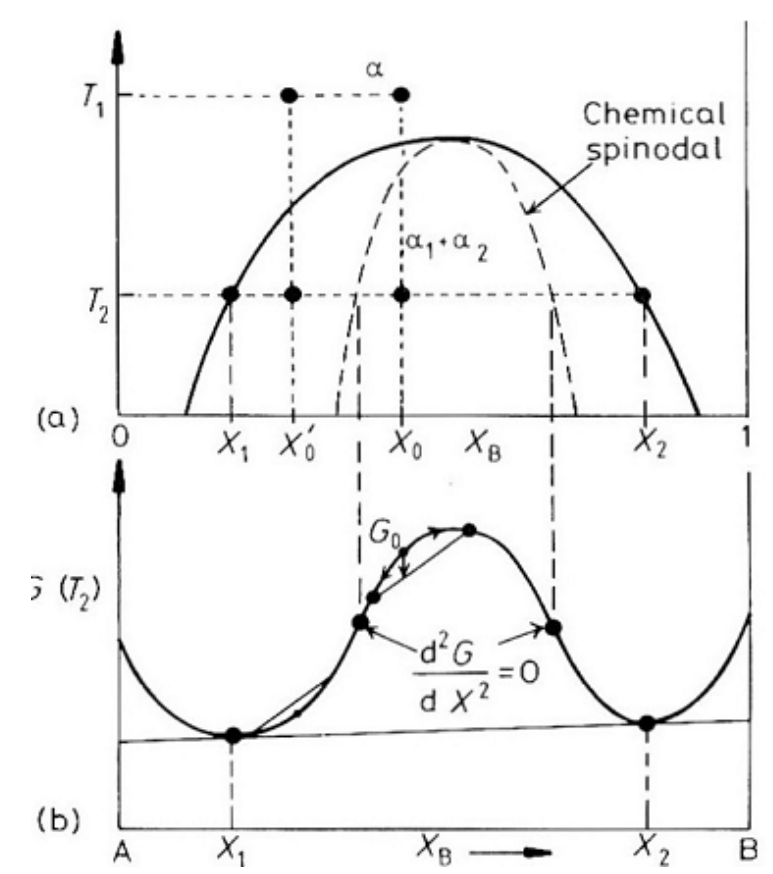

Figure 6. (a)Boldlinedesignatesthe (incoherent)miscibility gap and dashed line the (chemical) spinodal regions (b)

Gibbs energy-composition curve at $\mathrm{T} 2$

To enhance the mechanical properties of duplex stainless steel and to hinder the separation of gibbs free energy, we must raise the energy. The best treatment to hinder this separation is to apply magnetic field that can raise the thermal content of duplex stainless steel. We can use ferromagnetic material that has special movement in its magnetic domain like Nd2Fe14B. By its fractal movement, we can equalize and raise the gibbs free energy as $\mathrm{Nd} 2 \mathrm{Fe} 14 \mathrm{~B}$ is permenant magnet in it self (Kreyssig et al., 2009).

Also raising the gibbs free energy, give the austenitic stabalizers their chance to hinder the spinodal separation and hinder the chromium diffusion as we will discuss soonly.

Recently, many companies tend to manufacture manifolds in duplex stainless steel by HIP to enhance the mechanical properties of them.

The key word between the spinodal reactions, Nd2Fe14B and applied magnetic field is the manifold as the cahn hillard equations have special solutions in the manifold and Ising model has special holonomicity charachteristics on the manifold with the canonical singularities. We will show by category theory to establish mathematical relations between them.

By 1982 the latest version of the Fe-Cr phase diagram was that due to Kubaschewski, see figure 2.8a. Later on, Andersson and Sundman (Carpineti \& Giglio, 1992) optimizedall the available experimental data up to 1987 and constructed another version of the $\mathrm{Fe}-\mathrm{Cr}$ phase diagram. This phase diagram was rather differentfrom Kubaschewski's. However, these two did not issue any commentsonthespinodal line; this may be due to the fact that the spinodal is notreallypartofthephase diagram. Figures $2.8 \mathrm{a}$ and $\mathrm{b}$ also contain results of a study by Dubieland Inden (Roidos \& Schrohe, 2013) which was aimed at further characterization of the miscibilitygaplineaswellas eutectoid temperature of the reaction $\sigma \rightarrow \alpha+\alpha^{\prime}$. They performedlongterm annealing (2 to 11 years at 460, 500 and $510 \circ \mathrm{C}$ ) on Fe-Cr binaryalloysof $15,20,48$ and 70 at. $\% \mathrm{Cr}$ and determined the chemical compositionofaor $\alpha$ 'phases applying Mössbauer Spectroscopy (MS). Starting by a fully ferritic structure they observed that at $500 \mathrm{oC}$ no $\sigma$ phase was formed even after 4 years. 


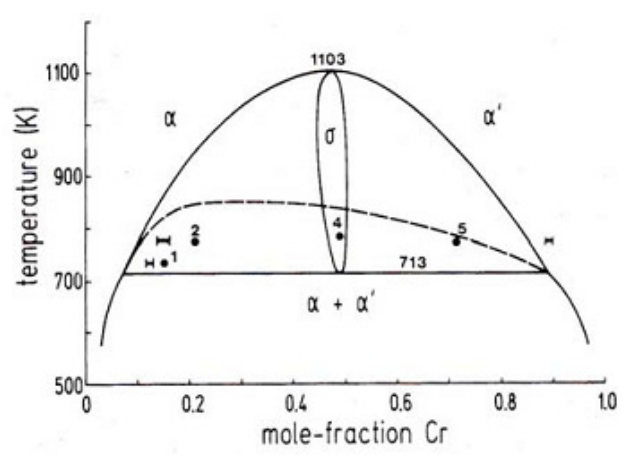

(a)

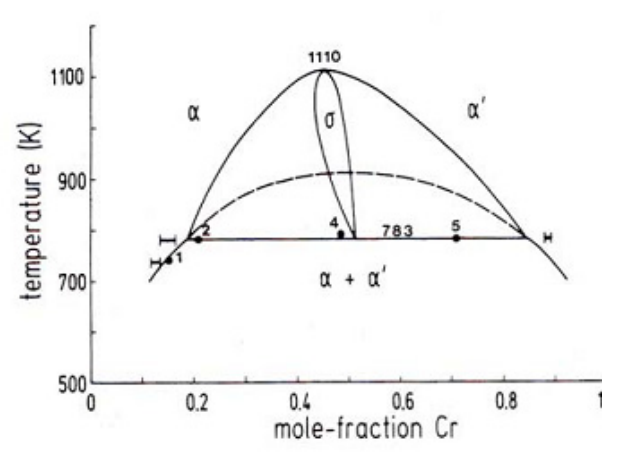

(b)

Figure 7. Fe-Cr phase diagram due to (a) Kubaschewski and (b) Andersson and Sundman. Numbers in the figures denote the initial composition of aged alloys by Dubiel and Inden. Horizontal bars correspond to the compositionrange of $\alpha$ and $\alpha^{\prime}$ phases measured by means of Mössbauer spectroscopy. These bars denote the miscibility gap boundary (Adams, Olubambi, Potgieter, \& Van Der Merwe, 2010; Kim et al., 2005; Udarne \& Nerjavnega, 2015; Maehara, Koike, Fujino, \& Kunitake, 1983; Park \& De Cooman, 2014; Frost \& Ashby, 1982)

Pattern formation processes due to phase separation of binary mixtures can conveniently be modelled by means of nonlocal Cahn-Hilliard equations. By this we mean $\mathrm{H}_{-1}$ gradient flows associated with functionals of GinzburgLandau type, which may include a dipolar interaction term. In a spatially periodic setting, i.e., using the three-dimensional torus $T=2 \pi R^{3} / Z^{3}$ as spatial domain, such functionals typically have the form

During the aging treatment, the spinodal nanostructure was formed in the $\delta$-ferrite. The spinodal structure would limit the lengths and movements of the dislocations during deformation. The function ghom(c) is the free energy per molecule of a homogeneous system of uniform concentration $\mathrm{c}$, which is non-convex in systems exhibiting phase separation. The gradient penalty tensor $\mathbf{K}$ is assumed to be a constant independent of $\mathbf{x}$ and $\mathbf{c}$. Then the diffusional chemical potential(in energy per molecule) is the variational derivative of Gmix (Burch \& Bazant, 2009)

$$
G_{m i x}[c]=\int_{V}\left[g_{\text {hom }}(c)+.5(\nabla c) \cdot(K \nabla c)\right] \rho d V
$$

The function ghom(c) is the free energy per molecule of a homogeneous system of uniform concentration c, which is non-convex in systems exhibiting phase separation. The gradient penaltyltensor $\mathbf{K}$ is assumed to be a constant independent of $\mathbf{x}$ and $\mathrm{c}$. Then the diffusional chemical potential(in energy per molecule) is the variational derivative of Gmix,

$$
\mu(X, T)=\frac{\partial g}{\partial c}-\nabla .(K \nabla c)
$$

The Cahn-Hilliard equation is a phase-field or diffuse interface equation which is mainly used to model phase separation of a binary mixture, e.g. a two-component alloy, but many other applications are encountered., one finds the equation stated in various forms. We shall consider here the version As usual, we model a manifold with conical singularities by a manifold with boundary $B$ of dimension $n+1, n \geq 1$, endowed with a conically degenerate Riemannian metric. On one hand, working on a manifold with boundary simplifies the analysis; on the other hand, the degeneracy of the Riemannian metric entails that geometric operators such as the Laplacian show the typical degeneracy they have on spaces with conic points in Euclidean space (Roidos \& Schrohe, 2013; Vertman, 2016; Bahuaud \& Helliwell, 2011). Permanent magnet is a ferromagnetic material that has a magnetization even if there is no external applied field. Using Ising model we can explain their properties, and the Ising model has special charchteristic in manifolds. Nd-Fe-B magnets has special properties, Fractal Magnetic Domains on Multiple Length Scales in Nd2Fe14BThe industrial strength ferromagnet, Nd2Fe14B has become a prototypical system for the study of magneticdomain structures. The Fractal movement is described by fameous Ginzburg Landau equations (Byrne, Spence, Olsen, Houghton, \& MCMAHON, 1994). 


$$
G(z(x))=G_{0}+\int_{W} G(z(x), \nabla z(\mathrm{x})) \mathrm{dV}
$$

where $F(Z(x), \nabla Z(x))$ is the free energy density; $\nabla Z=\partial Z / \partial x .$. For the Ginzburg-Landau potential, this density is $F$ $(\mathrm{Z}(\mathrm{x}), \nabla \mathrm{Z}(\mathrm{x}))=1$. Here $\mathrm{D}$ is a fractal mass dimension of the fractal medium, and $d V_{D}$ is an element of the D-dimensional volume:

$$
d V_{D}=C_{3}(D, x) d V_{3}
$$

The location of the transition is a function of the temperature, field amplitude and frequency. A finite-size scaling analysis of large-scale Monte Carlo simulations of the kinetic Ising model in an oscillatory field has shown that the dynamic phase transition is in the same universality class as the equilibrium Ising model. A result confirmed in a recent study of a time-dependent Ginzburg-Landau model in an oscillatory field. Pattern formation processes due to phase separation of binary mixtures can conveniently be modeled by means of nonlocal Cahn-Hilliard equations. By this we mean $H^{-1}$ gradient flows associated with functionals of Ginzburg Landau type, which may include a dipolar interaction term. Ohta-Kawasaki model, including the limiting case of $\sigma=0$ which corresponds to the classical Cahn-Hilliard model. However, the numerical method proposed here can be adapted to more general functions ${ }^{~} \sigma$ in (1.1), including, e.g., dipolar stray-field interaction in magnetic garnet films (Condette, Melcher, \& Süli, 2011)

$$
E(u)=\frac{1}{2} \int_{T^{3}} \varepsilon^{2}(\nabla u)^{2}+\frac{1}{2}\left(1-u^{2}\right)^{2} d x+\frac{1}{2} \sum_{k \in \mathbb{Z}^{3} /\{0\}} \sigma(k) u^{2}(k)
$$

Second, we shall consider $\frac{1}{2} \sum_{k \in z^{\prime}\{0\}} \sigma(k) u^{2}(k)=\sigma \int_{r^{\prime}}(\nabla \varphi)^{2} d x$ and $\mathrm{E}(\mathrm{u})$ becomes the Ohta-Kawasaki energy. One of the most remarkable and possibly the most accurate experimental verification for the universal glassy dynamics theoretically predicted for disordered elastic systems has been the measurement of the ultra-slow creep motion of magnetic domain walls in ultrathin. Nd-Fe-B magnets ferromagnetic films with perpendicular anisotropy driven by a very small (well below the depinning threshold defined below) applied magnetic fields. $V \sim \exp \left(-\frac{U_{c}}{k_{B} T}\left(\frac{H_{c}}{H}\right)^{\mu}\right)$, where Uc gives a characteristic energy scale in the creep regime and the applied field is

$H \ll H_{c}$ with $\mathrm{Hc}$ the so called depinning threshold. oth $\mathrm{Uc}$ and $\mathrm{Hc}$ are material dependent parameters and increase with the strength of the disorder. elastic manifold and its equilibrium roughness exponent $\zeta$ eq through the relation.

$$
\mu=\frac{d-2+2 \zeta e q}{2-\zeta e q} \text { (Frost \& Ashby, 1982). The roughness exponent } \zeta \text { eq measures the rate at which the interface }
$$

width grows with its linear size $\mathrm{w} \sim \mathrm{L} \zeta$ eqat equilibrium and is in turn universal: it depends only on $\mathrm{d}$, on the nature of the disorder correlations and on the short-ranged character of elastic interactions. This makes this experimental system a paradigmatic exampleof the universal physics predicted for elastic manifolds weakly pinned by random impurities. The theory for driven disordered elastic systems applied to magnetic domain walls also predicts two additional dynamical regimes as a function of H.26 For largefields H $\gg$ Hc (Vertman, 2016; Bahuaud \& Helliwell, 2011; Elliott \& Songmu, 1986)

In general permanent magnets are deeply explained by Ising model which exhibits high holonomicity behaviour under the scope of solobev spaces. Y. Yang generalized this definition to Finsler manifolds using Busemann volume form and Osculating Riemannian metric. We use this definition of Sobolev spaces in Finsler manifolds to prove two density theorems. Historically, one the significant density theorems is proved by S. B. Myers in 1954 for compact Riemannian manifolds and then in 1959 by M. Nakai for finite-dimensional Riemannian manifolds. Next in 1976 T. Aubin has investigated density theorems on Riemannian manifolds, Recently, in 2009 an extension of Myers-Nakai theorem to infinite-dimensional, complete Riemannian manifolds]. A similar result for the so-called finite dimensional Riemann-Finsler manifolds is given in in 2010. Next in 2011 the Myers-Nakai theorem is 
extended to the Finsler manifolds of class $C^{K}$.As usual, we model a manifold with conical singularities by a manifold with boundary $B$ of dimension $n+1, n \geq 1$, endowed with a conically degenerate Riemannian metric. On one hand, working on a manifold with boundary simplifies the analysis; on the other hand, the degeneracy of the Riemannian metric entails that geometric operators such as the Laplacian show the typical degeneracy they have on spaces with conic points in Euclidean space. We measure smoothness in terms of weighted Mellin-Sobolev spaces $\mathrm{H}_{p}^{s, \gamma}(\mathrm{B})$. Here $\mathrm{s}$ is a smoothness index, $\gamma$ a weight, and $1<\mathrm{p}<\infty$. They coincide with the usual $L^{p}$ (Roidos \& Schrohe, 2013; Vertman, 2016) -Sobolev spaces away from the singularities. As long as the integrands one considers are of a basically quadratic nature, hilbert manifolds of maps belonging to some Sobolev space $L^{p}$ are the natural manifolds in which to formulate the problems. However for more general integrands it becomes necessary to use Banach manifolds, for example manifolds of maps belonging to one of the more general Sobolev spaces $L^{p}$. Because of the volume-constraint, we shall be mainly concerned with situations where test functions have zero integralIn this case the following dual estimate will be crucial: if $u N \in X N$ and $v N \in X N$, their characterizing the homogeneous Sobolev-Slobodetski 1 space $\mathrm{H}^{\circ} \mathrm{s}(\mathrm{T} 3)$ as the closure, with respect to the induced homogeneous $H\left(\mathrm{~T}^{3}\right)$ we use the Yang's method to define certain Sobolev spaces onFinsler manifolds. Let dvF be the Busemann volume form, $\mathrm{f} \in C^{\infty}(\mathrm{M})$ and denote $L^{p}(M)=\{f: M \rightarrow R\}$ where $\mathrm{R}$ is measurable. Let us consider the vector space $\mathrm{C}(\mathrm{kp})$ of $\mathrm{C} \infty$ functions $\varphi$, such that $\nabla \phi \in L^{P}(M)$

Let $\mathrm{J}$ be a nonnegative, real-valued function, on the space of $C^{\infty}$ functions with compact support on IRn, denoted by $C^{\infty}(\mathrm{Rn})$ and having properties :

$$
\begin{gathered}
J(x)=0, \mathrm{x} \geq 1, \\
\int_{R^{m}} J_{c}(x) d x=1
\end{gathered}
$$

Chrome percentage varies with time of heating and also the magnetic domains have many fractal shapes. To emphaise conditions in all directions. $\lim D(b)=1$, for $\mathrm{K}<\mathrm{Kc}(\mathrm{T}>\mathrm{Tc}), D(b)=1.875$, for $\mathrm{K}=\mathrm{Kc}(\mathrm{T}=\mathrm{Tc})$, $\lim D(b)=2 \mathrm{~K} \geq \mathrm{Kc}(\mathrm{T}>\mathrm{Tc})$ is $J / k_{B} T,{ }^{b} \vec{k}_{B}^{\infty}$ is boltzman constant, Tis the temperature, where $\mathbf{J}$ defined by the following Hamilton (Falconer, 2004; Ito \& Suzuki, 1987). $H=-l / 2 ; S i S j(S i= \pm l)$. Also, heat kernels of dislocations on finsler manifolds details.
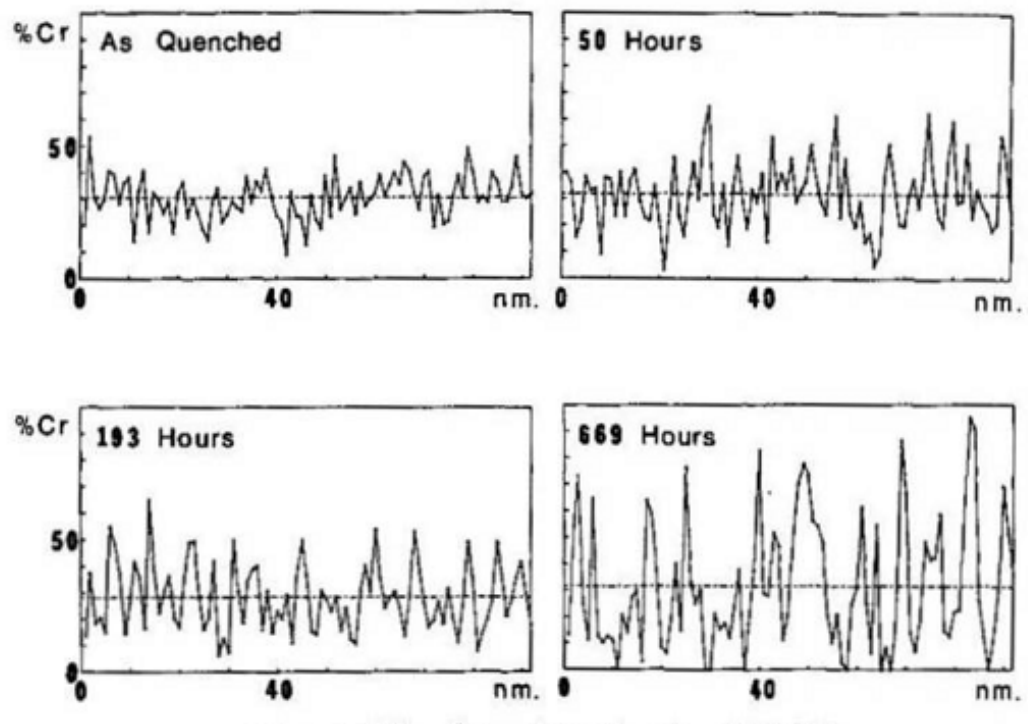

$\mathrm{Fe}-32 \% \mathrm{Cr}$ Aged at $470^{\circ} \mathrm{C}$

Figure 8. Concentration profiles of $\mathrm{Fe}-32 \mathrm{at} . \% \mathrm{Cr}$ samples in as-quenched and aged states. Numbers on the upper left of diagrams show the aging time in hours. Each data point in the diagrams corresponds to mean chemical composition of a volume element of the material $0.8 \mathrm{~nm}$ thick and $1 \mathrm{~nm}$ in diameter consisting of 30-50 atoms 

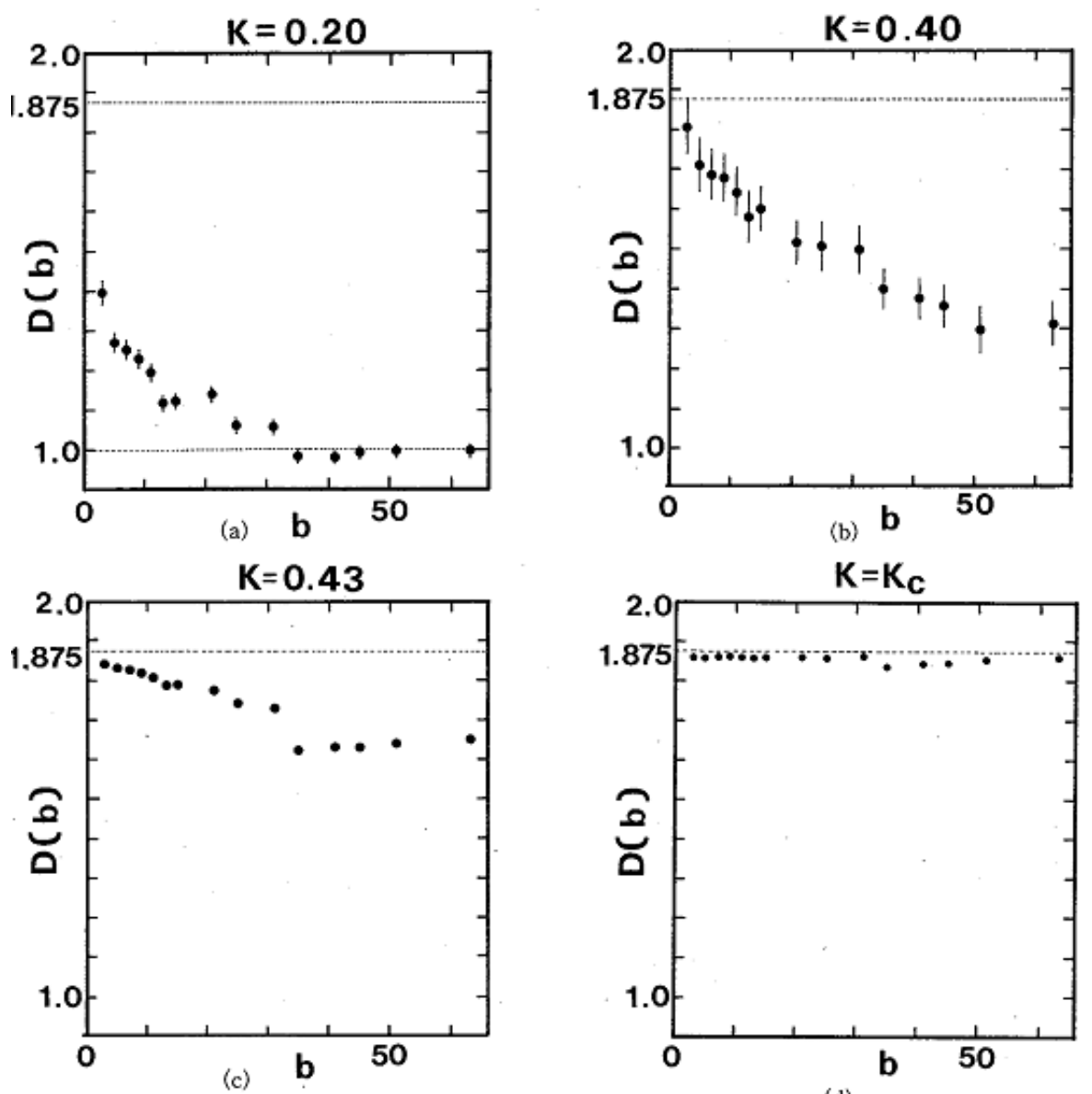

(d)

Figure 9. Values $\mathrm{D}(\mathrm{b})$ defined by equation(3) are shown as function of scale factor $\mathrm{b}$ for several temperatures. The system size $64 \times 64$ (a) $\mathrm{K}=.2$ when the sacle $\mathrm{b}$ is large the values $\mathrm{D}(\mathrm{b})$ approaches to unity, which corresponds to random percolation case. $(\mathrm{b}) \mathrm{K}=.4(\mathrm{c}) \mathrm{K}=.43(\mathrm{~d}) \mathrm{K}=\mathrm{kc}=.4407 \mathrm{D}(\mathrm{b})$ converges to the expected value 1.875 irrespective of the used scale factor (Ito \& Suzuki, 1987)

So, we can use the category theory to express about these finite objects as we have mentioned 4 shapes of fractals and four cr percentage with heating times. But the best way to regulate these parameters is to use the Lusternik-Schnirelman category as cahn hillard and magnetic fields are strongly related to solobev spaces as mentioned above.

DEFINITION. The Lusternik-Schnirelman category of A in X, cat(A: X), is the least integer $\mathrm{n}$ such that A can be covered by $n$ closed subsets of $X$ each of which is contractible in $X$. If no such integer $n$ exists we put cat $(A ; X)=$ $\infty$. We define $\operatorname{cat}(X)=\operatorname{cat}(X ; X)$

The Lusternik-Schnirelman category is used for finslers spaces especially. As we mentioned above, solobev space where Ising model and cahn hillard equations are highly related to it. Solobev space is suppoed to be defined on finsler manifold (Antonelli, Ingarden, \& Matsumoto, 2013; Bao, Chern, \& Shen, 1996).

MAIN THEOREM OF LUSTERNIK-SCHNIRBLMANTHEORY: Let M be a complete $C^{2}$ Finsler manifold without boundary, $f: M \rightarrow \mathbb{R}$ as $C^{2}$ map. Let $K$ denote the set of critical points of $f$ and for a $E \mathrm{R}$ let $K,=K$ of - '(a) and $M=f(-\infty, a)$. Assume for each a $E \mathrm{R}$ that a is not a limit point of int $f\left(k-k_{\alpha}\right)$

$$
c_{m}(f)=\operatorname{Inf}\left\{a \sim R \| \operatorname{cat}\left(M, ; M_{\alpha}\right) \geq m\right\} \text { then } c_{m}(f) \leq \mathrm{c}_{m+1}(f), 0 \leq m \leq n \leq \operatorname{cat}(M), c_{m}(f) \leq \infty \text { hence if }
$$

$M$ is connected then $\operatorname{dim} K_{c} \geq n-m$ (Bejancu \& Blair, 1990; Stanley, 2002). 
Gibbs free energy is hindered as it can not be used to express about all these parameters, so we the best solution is to use category theory.

So we can write the entire thermal content of the system as $C_{T}(M)=D(c(m)) E(\mathrm{c}(\mathrm{n}))$

$\mathrm{D}(\mathrm{c}(\mathrm{m}))$ is fractal measurement depends on $\mathrm{c}(\mathrm{m})$ as defined in main theorem of Lusternik Schnirbl main theory $\mathrm{E}(\mathrm{c}(\mathrm{n}))$ is dipolar field interaction depends on $\mathrm{c}(\mathrm{n})$ as defined in main theory of lusternik schinirby main theorem $C_{T}(M)$ is a thermal term of the entire system depends on fractal movement of magnetic domain as the morophology of the system depends on the applied magnetic field showed by figure (red spots) and also $C_{T}(M)$ depends on dipolar field interaction, it is a function in $\mathrm{M}$ finsler manifold as cahn hillard spinodal is related to manifolds with canonical singularities.

It has been showed the morophology of duplex stainless steel at aging in the case of application of high magnetic field figure.

\section{Conclusion}

By inserting Nd2FeB14 magnetic thin films in the HIP manifolds of duplex stainless steel, there will be enhancing in the mechanical properties of duplex stainless steel 2205 and the the impact energy will be raised to high levels. Impact energy will be enhanced as the spinodal reaction will be hindered. Pattern formation processes due to phase separation of binary mixtures can conveniently be modeled by means of nonlocal Cahn-Hilliard equations. By this we mean $\mathrm{H}^{-1}$ gradient flows associated with functionals of Ginzburg Landau type, which may include a dipolar interaction term. Ohta-Kawasaki model, including the limiting case of $\sigma=0$ which corresponds to the classical Cahn-Hilliard model. The Lusternik-Schnirelman category has been used to express about all the prameters and the objects which are used to enhance the mechanical properties of duplex stainless steel 2205 . The Lusternik-Schnirelman category is used to show how categories behave on finsler manifold. Finsler manifolds are strongly related to solobev spaces in which cahn hillard and Ising model show great charachterisics.

\section{References}

Adams, F. V., Olubambi, P. A., Potgieter, J. H., \& Van Der Merwe, J. (2010). Corrosion resistance of duplex stainless steels in selected organic acids and organic acid/chloride environments. Anti-Corrosion Methods and Materials, 57(3), 107-117. http://dx.doi.org/10.1108/00035591011040065

Akbar-Zadeh, H. (1988). Sur les espaces de Finsler à courbures sectionnelles constantes. Acad. Roy. Belg. Bull. Cl. Sci.(5), 74(10), 281-322.

Alvarez-Armas, I., \& Degallaix-Moreuil, S. (Eds.). (2009). Duplex stainless steels. John Wiley \& Sons.

Antonelli, P. L., Ingarden, R. S., \& Matsumoto, M. (2013). The theory of sprays and Finsler spaces with applications in physics and biology (Vol. 58). Springer Science \& Business Media.

Bahuaud, E., \& Helliwell, D. (2011). Short-time existence for some higher-order geometric flows. Communications in Partial Differential Equations, 36(12), 2189-2207.

Bao, D., Chern, S. S., \& Shen, Z. (1996). Preface for "Finsler geometry over the reals" (pp. 3-13).

Basyigit, A. B., \& Kurt, A. (2014). Corrosion Properties and Impact Toughness of 2205 Duplex Stainless Steel after TIG Welding*. Materials Testing, 56(10), 786-794. doi: 10.3139/120.110628

Bejancu, A., \& Blair, D. E. (1990). Finsler geometry and applications. New York: Ellis Horwood.

Bidabad, B., \& Shahi, A. (2013). On Sobolev spaces and density theorems on Finsler manifolds. arXiv preprint arXiv:1310.8027.

Borceux, F. (1994). Handbook of Categorical Algebra I: Basic Category Theory (Encyclopedia of Mathematics and Its Applications). Cambridge University Press.

Bucur, I., Deleanu, A., \& Hilton, P. J. (1968). Introduction to the Theory of Categories and Functors (Vol. 13, p. 13). London: Wiley.

Burch, D., \& Bazant, M. Z. (2009). Size-dependent spinodal and miscibility gaps for intercalation in nanoparticles. Nano letters, 9(11), 3795-3800. DOI: 10.1021/n19019787 •

Byrne, G., Spence, M. A., Olsen, B., Houghton, P. J., \& MCMAHON, J. (1994). Advantages of hot isostatic pressing (HIP) production routes for process manifolds, Duplex Stainless Steels' 94. In Proc. of Int. Conf. on Duplex Stainless Steels, Glasgow, Scotland, Cambridge TWI, paper (Vol. 19). 
Campbell, P. (1996). Permanent magnet materials and their application. Cambridge University Press. DOI: http://dx.doi.org/10.1017/CBO9780511623073

Carpineti, M., \& Giglio, M. (1992). Spinodal-type dynamics in fractal aggregation of colloidal clusters. Physical review letters, 68(22), 3327. DOI:10.1103/PhysRevLett.68.3327

Chiu, L. H., Hsieh, W. C., \& Wu, C. H. (2003). Cooling rate effect on vacuum brazed joint properties for 2205 duplex stainless steels. Materials Science and Engineering: A, 354(1), 82-91. http://dx.doi.org/10.1016/S0921-5093(02)00911-5

Coey, J. M. (2010). Magnetism and magnetic materials (pp. 128-174). Cambridge University Press. http://dx.doi.org/10.1017/CBO9780511845000

Condette, N., Melcher, C., \& Süli, E. (2011). Spectral approximation of pattern-forming nonlinear evolution equations with double-well potentials of quadratic growth. Mathematics of Computation, 80(273), 205-223.

Dos Santos, D. C., \& Magnabosco, R. (2016). Kinetic Study to Predict Sigma Phase Formation in Duplex Stainless Steels. Metallurgical and Materials Transactions A, 47(4), 1554-1565. http://dx.doi.org/10.1007/s11661-016 $-3323-\mathrm{z}$

Elliott, C. M., \& Songmu, Z. (1986). On the Cahn-Hilliard equation. Archive for Rational Mechanics and Analysis, 96(4), 339-357. DOI: 10.1007/BF00251803

Falconer, K. (2004). Fractal geometry: mathematical foundations and applications (pp. 44-66). John Wiley \& Sons. doi : 10.1002/0470013850

Frost, H. J., \& Ashby, M. F. (1982). Deformation mechanism maps: the plasticity and creep of metals and ceramics.

Hsieh, Y. C., Tsai, Y. T., Chang, Y. L., \& Yang, J. R. (n.d.). The interaction between dislocations and the spinodal nanostructure in a 2205 duplex stainless steel.

Huang, C. S., \& Shih, C. C. (2005). Effects of nitrogen and high temperature aging on $\sigma$ phase precipitation of duplex stainless steel. Materials Science and Engineering: A, 402(1), 66-75. http://dx.doi.org/10.1016/j.msea. 2005.03.111

Ito, N., \& Suzuki, M. (1987). Fractal configurations of the two-and three-dimensional Ising models at the critical point. Progress of theoretical physics, 77(6), 1391-1401.

Kangas, P., \& Newman, M. (1998). Performance of duplex stainless steels in organic acids. Anti-Corrosion Methods and Materials, 45(4), 233-242. http://dx.doi.org/10.1108/00035599810223698

Kim, S. C., Zhang, Z., Furuya, Y., Kang, C. Y., Sung, J. H., Ni, Q. Q., ... \& Kim, I. S. (2005). Effect of Precipitation of. SIGMA.-Phase and N Addition on the Mechanical Properties in 25Cr-7Ni-4Mo-2W Super Duplex Stainless Steel. Materials transactions, 46(7), 1656-1662.

Kreyssig, A., Prozorov, R., Dewhurst, C. D., Canfield, P. C., McCallum, R. W., \& Goldman, A. I. (2009). Probing fractal magnetic domains on multiple length scales in Nd 2 Fe 14 B. Physical review letters, 102(4), 047204.

Le Calvez, P. (2006). From Brouwer theory to the study of homeomorphisms of surfaces. In International Congress of Mathematicians (Vol. 3, pp. 77-98). doi:10.4171/022-3/4

Liu, X. Y., Xia, K. D., Niu, J. C., Xiang, Z., Yan, B., \& Lu, W. (2015). Effects of Heat Treatment on Microstructure and Pitting Corrosion Resistance of 2205 Duplex Stainless Steel. International Journal of Electrochemical Science, 10, 9359-9369.

Maehara, Y., Koike, M., Fujino, N., \& Kunitake, T. (1983). Precipitation of $\sigma$ phase in a 25Cr-7Ni-3Mo Duplex Phase Stainless Steel. Transactions of The Iron and Steel Institute of Japan, 23(3), 240-246. DOI: 10.2355/isijinternational1966.23.240

May, J. P. (1999). A concise course in algebraic topology. University of Chicago press.

Palais, R. S. (1966). Lusternik-Schnirelman theory on Banach manifolds. Topology, 5(2), 115-132. DOI: 10.1016/0040-9383(66)90013-9

Park, H., \& De Cooman, B. C. (2014). Creep Deformation of Type 2205 Duplex Stainless Steel and its Constituent Phases. ISIJ international, 54(4), 945-954. http://doi.org/10.2355/isijinternational.54.945

Phillips, N. S., Chumbley, L. S., \& Gleeson, B. (2009). Phase transformations in cast superaustenitic stainless steels. Journal of Materials Engineering and Performance, 18(9), 1285-1293. 
Pickering, F. B. (1976). Physical metallurgy of stainless steel developments. Int. Met. Rev., Dec. 1976, 21, 227-268.

Roidos, N., \& Schrohe, E. (2013). The Cahn-Hilliard equation and the Allen-Cahn equation on manifolds with conical singularities. Communications in Partial Differential Equations, 38(5), 925-943. DOI: 10.1080/03605302.2012.736913.

Rudraraju, S., Van der Ven, A., \& Garikipati, K. (2015). Mechano-chemical spinodal decomposition: A phenomenological theory of phase transformations in multi-component, crystalline solids. arXiv preprint arXiv: 1508.05930 .

Stanley, D. (2002). On the Lusternik-Schnirelmann category of maps. Canadian Journal of Mathematics, 54(3), 608-633. http://dx.doi.org/10.4153/CJM-2002-022-6

Udarne, P., \& Nerjavnega, I. D. (2015). Impact-Toughness Investigations of Duplex Stainless Steels. Materiali in tehnologije, 49(4), 481-486. doi:10.17222/mit.2014.133

Vertman, B. (2016). The biharmonic heat operator on edge manifolds and non-linear fourth order equations. Manuscripta Mathematica, 149(1-2), 179-203. 10.1007/s00229-015-0768-0

Warren, A. D., Harniman, R. L., Guo, Z., Younes, C. M., Flewitt, P. E. J., \& Scott, T. B. (2016). Quantification of sigma-phase evolution in thermally aged 2205 duplex stainless steel. Journal of Materials Science, 51(2), 694-707. http://dx.doi.org/10.1007/s10853-015-9131-9

\section{Copyrights}

Copyright for this article is retained by the author(s), with first publication rights granted to the journal.

This is an open-access article distributed under the terms and conditions of the Creative Commons Attribution license (http://creativecommons.org/licenses/by/4.0/). 